\title{
Community manager vs. social media manager Una delimitación teórica necesaria en el espacio comunicativo empresarial
}

\author{
José Sixto- García ${ }^{a *}$
}

aDoctor en Comunicación, Universidad de Santiago de Compostela; España. (ID

Forma de citar: Sixto, J. (2017). Community manager vs. Social media manager. Una Delimitación teórica necesaria en el espacio comunicativo empresarial. Perspectivas, 2(2). 95-107.

Recibido: febrero 12 de 2017

Aceptado: mayo 23 de 2017

\section{Palabras clave \\ Community manager, social media manager, dircom, reputación online, web 2.0.}

Resumen: En el momento en que surge la figura del community manager se entendió que su cometido debía consistir en gestión del contenido de los medios sociales. Sin embargo, en la medida en que la profesión se ha consolidado, van apareciendo otros perfiles como el social media manager. Con frecuencia se escuchan interpretaciones confusas acerca de ambas figuras, que en ocasiones se asimilan perfil y en funciones. En tal sentido, este artículo se propone describir con precisión qué es un community manager y qué debe entenderse por social media manager, cuáles son sus funciones específicas y por qué su contribución resulta imprescindible en la comunicación actual. Se aporta, asimismo, una interpretación fundamentada acerca de cómo deben integrarse en el organigrama organizacional y cuál debe ser su posición con respecto a la dirección de comunicación.

\footnotetext{
*Autor para correspondencia

jose.sixto@usc.es
} 


\section{Keywords}

Community manager, social media manager, dircom, online reputation, web 2.0.

\section{Palavras chave}

Community manager, social media manager, dircom, reputação online, web 2.0.

\section{Community manager vs. social media manager. A theoretical delimitation necessary in the business communication space}

\begin{abstract}
When the position of the community manager was established, it was understood that his/her role should be to manage a company or brand's social media content. However, inasmuch as the profession has been consolidated, other offices have appeared, such as the social media manager. There is frequent confusion in distinguishing between the two, who are sometimes assimilated in both profile and functions. Thus, this article aims to explain precisely what should be understood by both community manager and social media manager, as well as what their specific functions are and why their contribution is essential in present social media communication. It also provides a well-founded interpretation of how they should be integrated into the organization chart and what their position should be with respect to the office of communications.
\end{abstract}

\section{Community manager vs. social media manager. Uma delimitação teórica necessária no espaço de comunicação empresarial}

Resumo: No momento em que a figura do community managersurgiu, entendeu que seu papel deveria ser gerenciar o conteúdo das mídias sociais. No entanto, na medida em que a profissão foi consolidada, outros perfis apareceram, como osocial media manager. Freqüentemente, interpretações confusas são ouvidas sobre ambas as figuras, que às vezes são perfil e funções assimiladas. Nesse sentido, este artigo tem como objetivo descrever exatamente o que é um community managere o que deve ser entendido pelosocial media manager, quais são suas funções específicas e por que sua contribuição é essencial na comunicação atual. Ele também fornece uma interpretação bem fundamentada de como eles devem ser integrados no organograma e qual sua posição em relação à direção da comunicação. 


\section{Introducción}

Hace una década, casi nadie sabía lo que era un community manager (Meso, Mendiguren y Pérez, 2015), y en consecuencia, tal denominación no pertenecía al uso general, ni siquiera en el ámbito empresarial. Hoy en día el término se ha extendido incluso entre personas que no pertenecen al mundo de los negocios y la comunicación. Quienes se especializan en ellos, han oído el término cientos de veces, tanto en el ámbito académico como en el profesional, pero también, como se ha dicho, en situaciones y escenarios totalmente alejados de la comunicación, su estudio e investigación. Sin embargo, es probable que quienes conocen el término lo encuentren ambiguo, pues existen numerosas referencias e interpretaciones. Mucha gente, dentro y fuera de la academia y de los sectores profesionales, parece tener una opinión fundamentada sobre qué es un community management, pero las opiniones son dispares y el consenso, inexistente.

Incluso entre los propios profesionales que ejercen como community management, las explicaciones acerca de en qué consiste su trabajo a menudo difieren. Así, por ejemplo, para Borrás, community manager de El Ciruelo, "un buen community es aquel que es capaz de hacer que una marca pase a ser una persona física en la que confiar", mientras que para Gude, community manager de Leche Celta, "el community manager es la persona encargada de gestionar y dinamizar comunidades alrededor de una empresa o marca en las redes sociales y crear una imagen de esa marca en Internet" . Por su parte, Rodríguez, la community de la compañía de transportes terrestres de pasajeros Alsa, considera que se trata de "un profesional que escucha en el diván de Hootsuite y Google lo que se dice de una empresa, contacta con el responsable y da respuesta". Antoral, community manager de Antena 3, apela más directamente a la creación de una comunidad de usuarios en torno a una marca concreta y explica que "es el responsable de la comunidad online que se crea en torno a un producto o una empresa" (Russo, 2015).
Si la falta de unanimidad es patente en el entorno profesional, lo es más fuera de él. Por tanto, se hacen necesarios un análisis del estado de la cuestión y una revisión de las contribuciones académicas para determinar con exactitud cuál es el perfil profesional de esta nueva figura, ya imprescindible en el escenario comunicativo actual.

\section{Contexto comunicativo-empresarial para el surgimiento de un nuevo perfil profesional}

La presencia de las marcas en las redes sociales ha supuesto un cambio revolucionario en la comunicación y en el marketing. Hasta hace poco, la comunicación llegaba al consumidor de forma unidireccional a través de los medios masivos, pero ahora las posibilidades de interacción con las que cuentan los usuarios suponen una oportunidad para generar vínculos de afinidad y estrechar relaciones personalizadas con ellos y entre ellos mismos (Marhuenda y Nicolás, 2012).

En efecto, la comunicación organizacional también ha sido permeada por la llegada de Internet $\mathrm{y}$, específicamente, por la evolución de las redes sociales y los social media, que exigen gestión en un nuevo frente, la reputación digital. Es ahí donde se hace necesaria la presencia de un nuevo perfil profesional, el de la figura especializada en escuchar y dinamizar este tipo de comunidades sociales en entornos virtuales (Cobos, 2011). Este nuevo profesional es ahora responsable de sostener las relaciones de la organización en el ámbito digital, sobre la base del conocimiento de los planteamientos estratégicos de la entidad y los intereses de los públicos (Castelló, 2010). A día de hoy, tanto el perfil profesional como las funciones que ejerce se han consolidado y normalizado en el panorama de la comunicación corporativa $\mathrm{u}$ organizacional (Silva, 2016).

Pero, ¿qué consecuencias implica este nuevo escenario mediático para las organizaciones y cómo afecta a sus relaciones con los públicos? He aquí algunas consideraciones: (1) El nuevo entorno social permite a las audiencias comentar 
e interactuar sobre los acontecimientos al mismo tiempo que se producen. La audiencia también se ha vuelto social. (2) Se emplean diferentes pantallas o dispositivos para recibir un mismo contenido. Es decir, la interacción sobre un mismo hecho es múltiple a través de varios dispositivos empleados simultáneamente. (3) Los hechos y las noticias ya no solo se reciben; también se comentan, difunden y debaten en conjunto. Se pasa de la recepción individual al análisis en conjunto. Los receptores se convierten en distribuidores y difusores de contenido y los contenidos de base se alimentan con diversas aportaciones individuales o de grupo que los enriquecen. (4) Los receptores más colaborativos también crean contenidos. El medio es más de todos que nunca, aunque solo los periodistas continúan manteniendo la potestad para informar con rigor. (5) Medios y organizaciones son más democráticos que nunca. A los métodos de producción de noticias se han sumado las aportaciones sociales con las que los usuarios contribuyen a enriquecer los acontecimientos. Los usuarios no proporcionan noticias, sino hechos noticiosos que añaden valor al discurso, pero que pueden acabar transformándose en noticia. (6) Las técnicas de elaboración periodística han de ser más exhaustivas que nunca para discernir entre el rumor y la información. Ni todo lo que aportan los usuarios es verdad ni todo es mentira. Es ahí donde entra el papel del periodista y también en trasformar lo que puede ser un hecho noticioso en información contrastada. (7) Los medios sienten la necesidad de estimular la participación del usuario. El prestigio se pierde si no hay interacciones sociales, de tal manera que también se modifican los parámetros de medición de audiencias. Ya no solo importa cuántos leen o cuántos ven, sino cuántos impactos sociales genera esa recepción clásica. (8) La información es tremendamente actual, pues ahora todos tenemos capacidad de influencia sobre los contenidos en el mismo instante en que consideramos que algo ha cambiado. (9) Pueden generarse crisis en cuestión de segundos y con una capacidad de destrucción sobre la marca y la imagen con efectos devastadores.
Las empresas tienen facilidades para controlar y gestionar esas crisis al instante en que se están produciendo, pero los mecanismos de prevención y resolución tienen que ser más exhaustivos y estar preparados para poder usarlos con agilidad y precisión en cualquier momento. (10) Las redes sociales también son medio de comunicación. Gracias a la opción de compartir contenidos de medios tradicionales (fundamentalmente de los online) son el punto de acceso de muchos usuarios al medio oficial. Su utilización aumenta, por tanto, el tráfico web y el posicionamiento SEO de la página corporativa (Bellón y Sixto, 2015).

Así las cosas, podemos resumir el decálogo anterior en que la comunicación actual no puede entenderse únicamente desde una perspectiva 1.0. Por el contrario, a las organizaciones se les exige interacción y reciprocidad con los públicos, de modo que no se les permitiría que menospreciaran otros escenarios (más allá de los espacios físicos o los medios de comunicación) en los que sus públicos también están presentes y de los que son parte. Nos estamos refiriendo a todos los sitios surgidos a partir de la web 2.0, tales como las redes sociales, los blogs, las wikis, las webs corporativas interactivas, el posicionamiento en buscadores, los sistemas de geolocalización y/o mensajería instantánea, todos ellos caracterizados por su naturaleza bidireccional, por su inmediatez, por su recepción multiscreen, por poseer una concepción multimedia de base $\mathrm{y}$, en definitiva, por la posibilidad que ofrecen para crear conocimiento e inteligencia compartidos.

\section{Estado de la cuestión: situación actual del community management en las organizaciones}

Fruto de la revisión de la literatura académica y de la experiencia profesional en el sector se aprecia que las organizaciones no tardaron en darse cuenta de que si sus públicos habían ampliado sus escenarios de participación social, también ellas deberían encontrarse con clientes y usuarios en esos espacios de uso cotidiano. Conforme fueron interiorizando que ese nuevo modelo de comunicación no era 
anecdótico ni residual, también se percataron de que se precisaba un patrón de gestión. Desde un primer momento se entendió que quien debía asumir las responsabilidades comunicativas ligadas a estos entornos era una nueva figura profesional que se dio en llamar community manager.

Lo anterior no quiere decir que la problemática descrita esté ya completamente subsanada, ni que responda a cánones estratégico-organizativos ciento por ciento asentados, sino que tanto por la mocedad de estos sitios como por la transversalidad de los cometidos y de las funciones, así como por la habitual escasez de recursos humanos destinados a la gestión de la comunicación (sobre todo en PYMES), lo cierto es que todavía a día de hoy nos encontramos con cuatro situaciones diferentes de community management en función de las distintas organizaciones, según los resultados de este trabajo exploratorio:

a) Entidades con departamento de Comunicación en las que el dircom o su equipo se encargan de la gestión de los medios sociales, pudiendo haber una o varias personas destinadas en exclusiva a este cometido.

b) Entidades en las que se encargan de los medios sociales personas sin formación específica en la materia, incluso, a veces, sin ser expertos en Comunicación.

c) Entidades en las que la gestión de comunidades sociales es realizada por personas formadas en el área del community management, pero que no son periodistas ni poseen formación universitaria relacionada con la Comunicación.

d) Entidades que externalizan los medios sociales y confían estas tareas a una empresa externa o a un freelance que gestiona cuentas de distintas entidades.

e) Entidades que todavía no tienen presencia en medios sociales y desaprovechan, por tanto, las potencialidades que ofrecen estos sitios, acusando una carencia comunicativa muy grave.
Cuando la gestión de la comunicación 2.0 se realiza inhouse, dependiendo del tamaño de la organización y de los recursos humanos que compongan el gabinete de Comunicación y/o Marketing, se distinguen tres figuras profesionales competentes en la administración de los medios sociales:

\section{a) Community manager.}

b) Social media manager: se encargaría de la creación de las maniobras digitales de la entidad y del control de la estrategia y de las crisis de reputación, por lo que sus funciones tendrían un sentido creativo, analítico y de investigación, mientras que el community manager pasaría a estar bajo su supervisión y sería el encargado de ejecutar la estrategia que previamente habría diseñado el social media manager. En este sentido, las funciones del community manager se fundamentarían en controlar los medios sociales y publicar contenidos, hacer el seguimiento de la reputación y la imagen, así como crear y consolidar las comunidades de usuarios.

c) Dircom: puede ocurrir que sea el dircom, sobre todo en casos de gabinetes unipersonales, quien se ocupe de la gestión de los medios sociales. Se sobreentiende que tanto por su formación de base (graduado en Ciencias de la Comunicación) como por su reciclaje profesional $\mathrm{y} / \mathrm{o}$ formación complementaria podría asumir estas funciones con solvencia.

Partiendo de la idea de que las estrategias de comunicación social difieren en los escenarios, pero no en su esencia, que es la gestión de comunicación, entendemos que la gestión de los medios sociales debe estar bajo supervisión del dircom, como persona encargada de la gestión integral de la comunicación de la organización y que, por tanto, la comunicación (con independencia del canal por el que se traslade a los públicos) debe regirse por una política común, que es lo que dota de sentido la imagen corporativa organizacional. Esto no implica, sin embargo, que bajo la supervisión del 
social media manager o del community manager no puedan localizarse otros perfiles más especializados, que, incluso, dependiendo de la situación, podrían pertenecer a un departamento de Informática o Tecnología. A este respecto Battaglini (2015) afirma que el community es la voz de la empresa en las comunidades y la voz de las comunidades en las empresas pero que, desde su punto de vista, se limita a emprender acciones de marketing tradicional en redes sociales (tales como promociones, concursos o envío de muestras) y se pregunta qué es lo que, en verdad, cambiaba. Su contestación se fundamentó en la capacidad de responder a los usuarios a tiempo real.

\section{Discusión: ¿community manager o social media manager?}

\subsection{Justificación de la presencia del profesional comunicacional en las organizaciones}

Está claro que las organizaciones actuales tienen nuevas responsabilidades en materia comunicativa. La utilización de las herramientas tecnológicas que oferta el marketing digital tiene como finalidad promover cualquier tipo de producto (bien, servicio, idea, actividad, lugar, organización o persona) y son una arma poderosa en la gestión de la comunicación actual (Hernández, Silva y Rivera, 2013). Entre dichas herramientas destacamos: (1) definición/ participación en la construcción de la estrategia social de la organizacional, (2) actualización de los contenidos en medios sociales, (3) gestión de la reputación online, (4) investigación continua de la presencia de la competencia, (4) creación de cuadros de mando para la monitorización de resultados, (5) analítica de web social, (6) conocimiento de la organización en profundidad y (7) formación en actividades de inteligencia emocional de cara a la generación de diálogo social y consecución de engagement.

La llegada de las redes sociales ha propiciado la creación de nuevos puestos que amplía la demanda de trabajadores especializados por parte de las empresas (Silva, 2016). El community manager tiene a su cargo una parte fundamental de la comunicación organizacional: la comunicación digital en los escenarios digitales. Por ello, su trabajo nunca puede entenderse sin una coordinación exhaustiva con el dircom, puesto que la política comunicativa de toda entidad debe responder a los mismos parámetros estratégicos y a idénticos valores con independencia de cuál sea el canal o el soporte que se utilice para establecer contacto con los públicos. En consecuencia, su rol deberá centrarse siempre en la comunicación como punto de partida para la asignación de otros roles. (Rojas, 2011). Autores como Pérez, Gómez-Zorrilla y Marco (2015) hablan de la necesidad de emplear una herramienta de generación de reputación, de gestión de imagen de marca y un canal para comunicar la responsabilidad social corporativa, elementos que, junto con los asuntos públicos, las métricas y la propia comunicación, se consideran piezas claves para la consecución del éxito empresarial. Estos autores afirman que, como consecuencia, resulta indispensable introducir la Teoría de los Siete Pilares de la Comunicación Empresarial (respaldada por la consultora Lundquist) que en el informe de los 6th CSR On-line Awards indica que en un contexto donde los stakeholders demandan bidireccionalidad, transparencia y participación, un simple informe corporativo por parte de la empresa ya no es suficiente, sino que debe ir acompañado por una correcta estrategia de comunicación corporativa a través de los medios sociales que aporte a los mismos las herramientas de interactuación y participación más adecuadas. Tomando como modelo esta teoría los autores destacan la importancia de la diferenciación y la integración como áreas clave para conseguir el engagement de los stakeholders y sostienen la tesis de incluir un octavo pilar, la medición, ya que entienden que el análisis de las acciones es vital para llevar a cabo una buena actividad comunicacional y, por ende, de reputación corporativa. 


\subsection{El community manager}

Russo (2015) define el community manager como el profesional con conocimiento en las herramientas necesarias para hacer una buena gestión de las redes sociales de una empresa y que es el encargado de fomentar la comunicación e interacción de la organización con sus clientes, así como de monitorizar la presencia online de las marcas y de generar contenidos de valor capaces de mejorar la visibilidad de la empresa o entidad. De igual modo, establece que entre sus objetivos profesionales han de encontrarse los siguientes:

a) Mejorar la visibilidad de una marca o empresa en Internet.

b) Mejorar la reputación online de la marca.

c) Aumentar los seguidores en las redes sociales.

d) Mejorar el SEO de la empresa.

e) Mejorar el engagement entre la marca y la comunidad.

f) Prestar un servicio al cliente más personalizado. Esto implica, al mismo tiempo, mantener la percepción de la actividad de los usuarios e identificar a tiempo cualquier posible brote mediático a partir de un comentario negativo (Geifman, 2013).

g) Conseguir más suscriptores.

h) Identificar oportunidades de contenidos.

i) Conseguir establecer relaciones con influenciadores.

j) Maximizar las conversiones.

Ya en 2009 la Asociación Española de Responsables de Comunidad y Profesionales Social Media (AERCO-PSM) afirmó que el verdadero potencial del community manager radicaba en establecer una relación de confianza con la comunidad de usuarios o simpatizantes de la marca, recoger el feedback de los mismos y utilizarlo para proponer mejoras internas. En este sentido, establecía cinco tareas fundamentales en la labor específica de este profesional: a) Escuchar: monitorizar constantemente en Internet en busca de conversaciones sobre la empresa, los competidores o el mercado.

b) Hace circular internamente la información obtenida: a partir de la escucha debe ser capaz de extraer lo relevante, crear un discurso coherente y comprensible y hacerlo llegar a los departamentos o a las personas correspondientes dentro de la organización.

c) Explicar la posición de la empresa a la comunidad: el community manager es la voz de la empresa hacia la comunidad, en este sentido, es una voz positiva y abierta que traduce el discurso interno de la organización a uno comprensible para los usuarios. Responde y conversa activamente en todos los social media en los que la empresa tiene presencia activa o en los que se produzcan menciones relevantes. Asimismo, escribe para el blog corporativo u otros espacios de los medios sociales, usa todas las posibilidades multimedia a su alcance, y selecciona y comparte contenidos de interés para la comunidad.

d) Detectar los líderes de opinión o influenciadores: la relación entre la comunidad y la empresa está sostenida en la labor de los líderes o influencers del momento y los potenciales. El community manager debe ser capaz de identificarlos y reclutarlos tanto dentro como fuera de la organización.

e) Encontrar vías de comunicación entre la comunidad y la empresa: el community manager topará caminos y ayudará a diseñar estrategias de colaboración a los directivos de la entidad que desconocen cómo la comunidad puede ayudar a hacer crecer la empresa.

Ponte (2010) especifica que las funciones del community manager no suponen una novedad, ya que tradicionalmente las han venido realizando otras áreas de la empresa de forma offline, solo que ahora hay herramientas para hacerlo de modo online. Por su parte, Piñeiro (2010) señala doce tareas que ejecuta el community manager en el seno de la organización: (1) estar, ante todo, atento a los 
intereses de la firma, no se le debe escapar nada que sea útil para la empresa; (2) establecer qué canales de comunicación se van a utilizar (elegir aquellos más efectivos para llegar eficazmente a la comunidad); (3) mantener una comunicación fluida con otras áreas de la empresa (Informática, Producción, Marketing, Comercial, Desarrollo, etc.); (4) estar al tanto de todo lo que se dice acerca de la empresa y de la marca en Internet y su trascendencia a los medios de comunicación tradicionales; (5) estar igualmente al tanto de todo lo que se dice de la competencia y de lo que la competencia hace; (6) utilizar herramientas de seguimiento y monitorización, analizar la información obtenida y proponer estrategias y nuevas acciones; (7) tener criterio para, de entre todos los comentarios publicados, destacar aquellos positivos, negativos o notables, que por alguna razón merezcan la ejecución de alguna estrategia especial (estrategia que será sugerida y diseñada seguramente por otro departamento, por ejemplo, si se sugiere un fallo del producto, será Producción el departamento indicado de elaborar un plan de contingencia); (8) saber detectar las personas más proactivas entre todas con las que establece comunicación, puesto que una persona proactiva, también denominada líder de opinión, supone una gran ganancia de tiempo a la hora de alcanzar los objetivos propuestos; (9) ser resolutivo, es decir, debe ser el tipo de persona útil con la cual toda empresa sabe que puede contar y todo cliente sabe que es mejor preguntarle a ella porque genera respuestas útiles. Por ello debe hacerse conocer en las comunidades online donde es activo y ayudar a los usuarios y posibles clientes a resolver cualquier conflicto mostrándose como un nexo entre la empresa o marca y el usuario; (10) ser rápido en la resolución de conflictos, ya que si un conflicto tarda un día en resolverse probablemente se trate de un conflicto no resuelto y en muchos casos una crisis fuera de control que propiciará una fuga de clientes hacia la competencia, afectando igualmente a la reputación online; (11) el community manager no vende ni hace publicidad; el community manager comunica y resuelve; y, finalmente, (12) debe informar a los usuarios de toda acción que lleve a cabo la empresa, lo que supone que ha de ser una persona que aplique la logística a la comunicación dentro y fuera de la organización.

Para Martínez (2013) el community manager es el profesional responsable de construir, gestionar y administrar la comunidad online alrededor de una marca en Internet, creando y manteniendo relaciones estables y duraderas con sus clientes, sus fans y, en general, con cualquier usuario interesado en la marca. Desde este punto de vista, Martínez recalca que la gestión de una marca en Internet exige poseer un conjunto de habilidades, técnicas y destrezas que es necesario adquirir y potenciar, para lo que el autor aconseja programas formativos en community management.

El desarrollo y aparición de una amplia variedad de herramientas en Internet ha supuesto el crecimiento de las redes sociales, que aumenta ofreciendo múltiples posibilidades de comunicación e interacción (Infante y Aguaded, 2012). En un entorno empresarial en el que la interactividad, la proactividad y el usuario son los factores fundamentales del éxito de la mayoría de las estrategias de marketing es imprescindible tener claro cómo situar la empresa en la web 2.0 (Cortés y Martínez, 2010). De todas formas, debido a la constante evolución tecnológica y a la permanente actualización de soportes móviles y multimedia, no es fácil predecir cómo van a evolucionar los cometidos inherentes al trabajo y a las funciones de un community manager. Con todo, desde el blog del Grupo IOE (2015) se indican siete claves para desarrollar con éxito la práctica profesional en 2016: (1) Un calendario con las fechas importantes sobre las publicaciones de campañas, las ruedas de prensa, los días claves donde se tenga que hacer un pequeño comunicado, etc. (2) Ser preventivo: todo community debe estar al tanto de lo que es noticia y llevarlo a su campo para informar a los usuarios de curiosidades o poder enlazarlo con la marca de la empresa, de tal modo que se ofrezcan contenidos variados y que no saturen al usuario. (3) Saber cómo comunicar, es decir, identificar el mensaje y concretar con qué 
herramienta se va a transmitir, teniendo siempre en cuenta las particularidades propias de cada red o medio social. (4) El seguimiento es importante: el community no debe dejar sin supervisar cualquier ninguna interacción, lo que le permitirá realizar un análisis de las horas a las que las publicaciones son más exitosas, qué numero de clics reciben, etc. (5) No preocuparse ante la falta de ideas y apostar siempre por la creatividad. (6) El feedback: el community ha de ser prudente a la hora de contestar y tener en cuenta que representa a una organización, por lo que la respuesta se sobreentiende que se produce en nombre de la entidad y no personalmente. (7) Informarse a todas horas de noticias de actualidad, en especial de noticias relacionadas con el sector profesional de la organización.

\subsection{El social media manager}

En años anteriores se decía que el community manager era la voz de la empresa en las comunidades y la voz de las comunidades en las empresas pero, según las aportaciones de Battaglini (2015), se limitaba a hacer acciones de marketing tradicional en redes sociales: promociones, concursos, envío de muestras. Tras la aparición e incipiente consolidación de la figura del community manager se empezó a hablar de otro nuevo perfil profesional: el social media manager (SMM). Situado en un puesto superior en el organigrama organizacional. Dn efecto, el SMM se encargaría de la creación de las estrategias digitales de la entidad y del control de la estrategia y de las crisis de reputación, por lo que sus funciones tendrían un sentido más creativo, analítico y de investigación. El community manager, por su parte, pasaría a estar bajo la función del SMM y sería el encargado de ejecutar la estrategia que el SMM previamente habría diseñado, de modo que sus tareas se fundamentarían en el control de las redes sociales y la publicación de contenidos, el seguimiento de la reputación y la imagen, y la creación y consolidación de las comunidades.

Es lógico que SMM y dircom deban trabajar mano a mano, pues ambos se encargan de la gestión integral de comunicación: el primero en el ámbito digital y el segundo en el tradicional. Las estrategias de comunicación social difieren en los escenarios, pero no en su esencia, que es la gestión de comunicación, por lo que entendemos que deben estar bajo supervisión del dircom, en tanto responsable de la gestión integral de la comunicación de la institución y que, por tanto, la comunicación, con independencia del canal por el que se traslade a los públicos, debe regirse por la misma política y sustentarse en una coherencia, una cohesión y una ejecución temporal que doten de sentido la imagen e identidad corporativas de la entidad en su conjunto.

El perfil del social media manager adquiere una importancia máxima cuando hablamos del tratamiento y la administración de las comunidades de marca, entendidas como la última etapa del marketing relacional, en el que cada vez más se potencia una interacción one to one y una comunicación personalizada hasta el extremo (Sixto, 2015). Estas comunidades de marca son, en realidad, grupos de personas con un interés compartido por un producto determinado, lo que condiciona que la marca se sitúe en un lugar clave. Las comunidades de marca se extienden más allá del marketing de relaciones, puesto que los motivos que justifican su existencia abarcan la relación entre quien consume y quien ofrece, de tal forma que surge un sentimiento de fidelidad social hacia la propia comunidad de usuarios que no concibe bajo ningún parámetro lo que podría entenderse como una deslealtad hacia un modo de vida que es cooperado (Saavedra, Linero y Gutiérrez, 2011).

Las relaciones basadas en la web son un medio para llegar a un fin (encontrar aliento, apoyo emocional, sentirse vinculado entre iguales) y no un fin en sí mismo (ganar estatus por el uso de la marca), por lo que la consistencia de los mensajes se convierte en la herramienta fundamental para que los usuarios puedan alcanzar sus objetivos personales a través de la marca (Fournier y Lee, 2009), cumpliendo así sus expectativas y acumulando experiencias. Castelló (2010) considera que algunas de las estrategias organizacionales que se puede poner en marcha 
mediante la explotación de este tipo de comunidades son: (1) Orientación hacia el cliente. La web 2.0 ha permitido el paso de una economía de mercado a una economía de las relaciones en la que el cliente es la principal variable de marketing que guía la toma de decisiones. Las marcas buscan estrategias para conseguir el nivel más alto de lealtad del cliente como paso previo a la fidelización. (2) Segmentación. Las posibilidades de afinidad para construir el vínculo entre marca y usuario que ofrece Internet favorece a la organización el establecimiento de relaciones individualizadas. La segmentación de mercado pone de manifiesto las oportunidades de negocio existentes al identificar grupos con demanda desatendida, contribuye a establecer prioridades, facilita el análisis de la competencia, favorece el ajuste de la oferta a necesidades específicas y permite optimizar la inversión publicitaria. (3) Viralidad. La recomendación de conocidos es el canal en el que más confían los consumidores en sus decisiones de compra y consumo. (4) Branding. Las comunicaciones personalizadas, las interacciones y las experiencias de marca que pueden ofrecerse en los medios sociales facilitan la creación de comunidades en torno a la marca y, por lo tanto, generan branding social.

\section{Resultados: propuesta de organigrama organizacional para departamentos de Comunicación}

Aun considerando las figuras de community manager y social media manager, el organigrama organizacional en materia comunicativa debería presentarse con el dircom como responsable último de la política comunicativa de la entidad y, jerárquicamente por debajo, el SMM como responsable último de la comunicación en medios sociales, entendiendo por ellos no solo las redes sociales, sino también los blogs y sus variantes, las utilidades gráficas o las wikis, tal y como se aprecia en la figura 1:
Figura 1

Organigrama corporativo del departamento de Comunicación

\section{Dircom}

Social media manager

\section{Community manager}

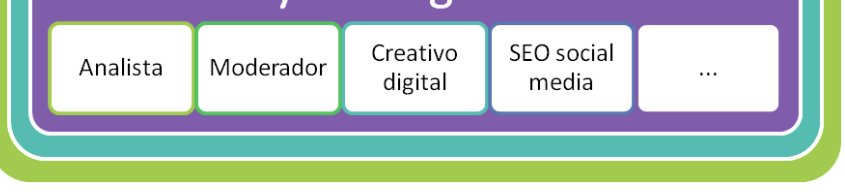

Fuente: Autor, Proceso de investigación.

Estarían bajo supervisión del SMM los nuevos perfiles profesionales que han ido surgiendo en estos últimos años vinculados a la especialización en los medios sociales, esto es, el community manager como el creador de contenidos, el analista web como el evaluador de la reputación, el social media SEO como el adaptador de los contenidos para su mejor posicionamiento en buscadores, el moderador de debates en páginas o grupos y, así, otros perfiles que pudieran concebirse en función del tamaño de la organización o del número de personas dedicadas a estos cometidos. Crucianelli (2010), por ejemplo, diferencia entre social media editor, community manager, reportero wifi, reportero multimedia, data base finder, gestor adSense, administrador de blogs, ejecutivo business y ejecutivo en alianzas. Por su parte, Correyero y Baladrón (2010) apuntan a la presencia de la figura del community manager y aluden a otras figuras profesionales como social media manager, news developer, keyword manager, search editor, newsroom technology manager y SEO (search engine optimizer). En una aportación semejante Vinader, Abuín y García (2011) señalan la presencia del community manager y del SEO, pero añaden social media analyst, chief blogging officer y creativo digital.

No obstante, el organigrama presentado responde a un planteamiento académico y purista, concebido bajo una perspectiva investigadora y 
quizás demasiado teórica y poco aplicada en estos momentos. La propuesta es todavía demasiado idealista y solo existe en grandes compañías o en instituciones que cuentan con departamentos de Comunicación integrados por varios equipos o personas. La realidad común es muy diferente. Son muchas las empresas (e incluso instituciones públicas) que ni siquiera tienen un departamento de Comunicación y muchas también las que poseen uno unipersonal, lo que significa que en esas situaciones un único sujeto se encarga de la gestión comunicativa en todas las esferas en las que la organización tiene presencia.

En consecuencia, a día de hoy es válido emplear community manager para describir a la persona encargada de la gestión integral de las comunidades sociales de una organización. Más todavía, en la actualidad se trata del concepto mayoritariamente utilizado y el que todavía se está asentando en los modelos de gestión presentes. Con todo, lo que sí es preciso recalcar de nuevo es que el community debe realizar sus funciones siempre bajo la supervisión del dircom, que es, en definitiva, el encargado de la comunicación, y que una discordancia entre ambo puede provocar, por ejemplo, que se difunda una información antes en las redes sociales que en la rueda de prensa convocada, por lo que esta última carecería de sentido.

\section{Conclusiones y propuesta de futuro}

Los medios sociales son herramientas de comunicación que exigen estrategias de gestión, al igual que ocurre con los medios tradicionales. En este sentido, la gestión de medios sociales debe atender tanto los públicos internos como externos de la organización.

La gestión de la comunicación exige la adaptación de los mensajes tradicionales a los nuevos medios atendiendo a sus formas y modelos de redacción y al seguimiento de un protocolo de actuación para la gestión de tiempos, así como de rutinas profesionales propiciadas por los cambios tecnológicos. En efecto, la política global de comunicación ha de contemplar estos espacios y mantener y explotar en ellos una imagen y una identidad corporativas acordes con las de la organización en su totalidad. Por tanto, comunicación offline y online deben responder en su filosofía a los mismos objetivos y mantener una línea editorial idéntica, a pesar de que las organizaciones hayan ampliado sus públicos y los segmentos sean ahora más concretos y más definidos.

La figura profesional encargada de la gestión de estos nuevos medios suele denominarse community manager. Aunque se trata de una nomenclatura correcta, debido a la novedad de las tecnologías y a las situaciones variopintas que reproduce en este caso el panorama mediático actual, sobre todo derivadas del tamaño de la organización, se contemplan otros perfiles como el social media manager. Desde una posición superior en la jerarquía empresarial el social media manager planificaría la estrategia y el community la pondría en práctica, lo que quiere decir que el primero idea y el segundo opera. En cualquier caso, ambas figuras deben regirse por las líneas estratégicas en materia comunicativa que se hayan planificado desde la dirección de Comunicación, puesto que los medios sociales son sociales, pero también son medios.

Con independencia de las diversas situaciones particulares que puedan producirse, lo que está claro es que alguien debe gestionar la comunicación digital en espacios sociales, pues todas las organizaciones y marcas suman a su reputación tradicional la reputación online, surgida a partir de la percepción que tiene el público de la organización y del prestigio que le otorga en la red. En la sociedad digital del conocimiento, la información ha dejado de ser bien de consumo para convertirse en la clave del progreso de las personas y las colectividades. La gestión del conocimiento se ha transformado para que las instituciones y empresas puedan sobrevivir dignamente en la sociedad digital (Ortega y Ortega, 2013). Se trata, en definitiva, de una unión racional y emocional entre la organización y sus partícipes graduada por la capacidad organizacional para satisfacer las necesidades de los públicos. Por ello, 
a continuación se destacan como resumen cuatro etapas fundamentales que ninguna organización actual puede desatender y que, como es lógico, sebe encargar a alguien de su gestión:

a) Etapa 1: Escuchar mediante la investigación y la monitorización el diálogo online, ya que Internet nos ofrece la oportunidad de conocer exactamente qué, quién y dónde está hablando de la marca.

b) Etapa 2: Valorar las opiniones. Una vez rastreadas las opiniones y los comentarios sobre la organización y su(s) marca(s) es hora de analizar la información recopilada. Se ha de valorar cada una de las opiniones que se han monitorizado, analizar tendencias y ponderar el total de menciones. La valoración de la reputación online tiene que responder a tres preguntas: ¿Qué se está diciendo? ¿Quién lo está diciendo? ¿Dónde se está diciendo?

c) Etapa 3: Monitorizar y valorar la reputación de la competencia. Examinar la competencia permite valorar la reputación propia en términos relativos a un sector específico.

d) Etapa 4: Participar. La actitud del social media manager o del community manager nunca puede ser pasiva. Es más, no basta con que sea activa, sino que debe ser colaborativa, esto es, debe participar activamente en red, pero además debe crear contenido. Distinguimos, pues, dos tipos de actitud:

- Actitud reactiva: Identificadas las opiniones, lo aconsejable es participar siempre, tanto si las opiniones son positivas como negativas. Solo actuando sobre la causa que ha motivado la creación de una determinada reputación online se estará haciendo bien los deberes a largo plazo. Las opiniones negativas no deben preocupar en exceso ni identificarse como un problema de facto, ya que en muchas ocasiones sirven de feedback para mejorar y potenciar aún más la reputación positiva de la organización.

- Actitud proactiva: No basta con escuchar y analizar, hay que tomar la iniciativa. Estos son algunos ejemplos que ayudan a la consolidación de una estrategia audaz: (1) Revisión del sitio web para verificar que las páginas se están indexando y posicionando en los buscadores para términos relevantes. (2) Creación de micrositios adicionales con contenidos que potencien una reputación positiva. (3) Creación y mantenimiento de un blog corporativo temático y especializado. (4) Mantenimiento avivado de una presencia activa tanto en redes sociales horizontales como verticales en función de la estrategia predefinida. (5) Inserción de la organización en directorios sectoriales de calidad y en directorios 2.0.

\section{Referencias}

Battaglini, M. (2015). La figura del Community manager: de la moda a la consolidación. Anuncios: Semanario de publicidad y marketing, $1512,34$.

Bellón, A. y Sixto, J. (2015). El uso de las redes sociales por 20 Minutos, único superviviente del modelo de prensa de distribución gratuita en España. Actas del I Simposio de la Red Internacional de Investigación de Gestión de la Comunicación (XESCOM, 2015), 908-922.

Castelló, A. (2010). Una nueva figura profesional: el Community manager. Pangea: Revista de la Red Académica Iberoamericana de Comunicación, 1, 74-97.

Cobos, T. L. (2011). Y surge el Community manager. Razón y Palabra, 75. Disponible en: $<$ http://www. redalyc.org/articulo.oa? $\mathrm{id}=199518706051>$ ISSN 1605-4806

Correyero, B. y Baladrón, A. (2010). Nuevos perfiles profesionales en el entorno digital: un desafío para la formación de comunicadores desde el EEES. Actas del II Congreso Internacional Latina de Comunicación Social, Tenerife: Universidad de La Laguna.

Cortés, M. y Martínez, Ch. (2010). El nuevo marketing y la figura del community manager. Harvard Deusto Marketing y Ventas, 16-24.

Crucianelli, S. (2010). Digital tools for journalists. Knight Center for Journalism in the Americas: 
University of Texas.

Fournier, S. y Lee, L. (2009). Creating Good Brand Communities. Harvard Business Review, 87(8), 113-122.

Geifman, A. (2013). Community Managers. El secreto detrás del éxito de social media. Revista Merca 2.0, 27-31.

Grupo IOE (2016). 7 Community manager keys for 2016. Retrieved from http://blog.ioe.es/7claves-community-manager-2016/

Hernández, Á.; Silva, D. y Rivera, E. (2013). El community manager: características y funciones básicas. Revista de Psicología y Ciencias del Comportamiento, 4(2), 67-75.

Infante, A. y Aguaded, J. I. (2012). Las redes sociales como herramientas educativas. Las tecnologías de la información en contextos educativos: nuevos escenarios de aprendizaje, 163-176.

Marhuenda, C.y Nicolás, M.A. (2012). Herramientas para la medición de los social media. En Nicolás M. A. y Grandío M. M. (coords.). Estrategias de Comunicación en Redes Sociales. Usuarios, aplicaciones y contenidos, 31-50.

Martínez, E. (2013). Qué es un Community manager y cuáles son sus principales funciones en la empresa. Disponible en http://comunidad. iebschool.com/iebs/general/que-es-uncommunity-manager/

Meso, K.; Mendiguren, T. y Pérez, J. (2015). The role of community manager in the marketing strategies of the Spanish companies most active on social networks. Estudios sobre el mensaje periodístico, 21(1), 385-402.

Ortega, J. A. y Ortega, Á. (2013). El perfil del Community manager en Entornos Educativos. Creatividad y sociedad: Revista de la Asociación para la Creatividad, 21.

Pérez, C.; Gómez-Zorrilla, J.M. y Marco, J. (2015). La comunicación empresarial en la Web 2.0. Estrategias para la gestión efectiva de la reputación corporativa. TyCE, Tecnología, Ciencia y Educación, 1, 62-67.

Piñeiro, G. (2010). Community manager. Definición, funciones, tareas y perfil.
Disponible en http://www.elblogdegerman. com/2010/11/09/community-managerdefinicion-funcionestareas-y-perfil-smo/

Ponte, D. (2010). Community manager, ¿una profesión con fecha de caducidad? Disponible en http://www.marketingcomunidad.com/ community-manager- $\% \mathrm{C} 2 \% \mathrm{BFuna}$-profesionconfecha-de-caducidad.html

Rojas, P. (2011). Community Management en una semana. España: Gestión 2000.

Russo, C. (2015). Qué es un Community manager. La guía jamás contada. Disponible en http:// claudioinacio.com/2015/09/08/que-es-uncommunity-manager/

Saavedra, J. L.; Linero, O. R. y Gutiérrez, R. (2011). Community manager y la marca como estrategia organizacional en las redes sociales online, REDHECS: Revista electrónica de Humanidades, Educación y Comunicación Social, 10, 168-181.

Silva, C. (2016). Perfil del community manager en las agencias de publicidad y relaciones públicas de España. El profesional de la información, 2(25), 237-245.

Sixto, J. (2015). Desarrollo de las redes sociales como herramienta de marketing. Estado de la cuestión hasta 2015. Anagramas, 13(26), 179196.

Vinader, R., Abuín, N. y García, A. (2011). Competencias digitales. El impacto de la Web 2.0 en los perfiles profesionales del sector audiovisual. Telos, 87, 78-83. 